\title{
Evaluation of a rapid method for identifying Branhamella catarrhalis
}

\author{
J RICHARDS From the Public Health Laboratory and Department of Microbiology, Leicester Royal \\ Infirmary, Leicester
}

SUMMARY The speed and precision of hydrolysis of tributyrin to butiric acid as a test to detect Branhamella catarrhalis were evaluated. The test proved consistently reliable in the identification of strains and correctly differentiated $B$ catarrhalis from Neisseria sp. The combination of Gram stain, oxidase, and catalase tests to tributyrin hydrolysis provides a means of positive same day identification of $B$ catarrhalis.

Branhamella catarrhalis has long been regarded as a harmless commensal of the upper respiratory tract; it is only in recent years that its pathogenic role in lower respiratory tract infection in patients with chronic chest disease, otitis media in children, and in sinusitis in all age groups has been recognised..$^{1-3}$ The high proportion of isolates producing $\beta$ lactamase is also a cause for major concern as this increases the potential of $B$ catarrhalis to act as a secondary pathogen. ${ }^{45}$

A prompt and reliable means of identification following isolation from clinical specimens is therefore required: the criteria enumerated by Doern and Morse in $1980,{ }^{6}$ although useful and reliable, have proved cumbersome for the routine busy diagnostic microbiology laboratory. Most workers have relied on colonial appearance, Gram stain, and a set of negative reactions in sugar media to differentiate $\boldsymbol{B}$ catarrhalis from Neisseriaceae ( $N$ meningitidis, $N$ gonorrhoeae, and $N$ lactamica) present in the upper respiratory flora. Rioux et al $^{7}$ described hydrolysis of tributyrin to butiric acid as one of the key characteristics in the identification of Branhamella sp. The method uses commercially available tablets impregnanted with tributyrin (LAB M, Topley House, Bury, Lancashire).

\section{Material and methods}

Forty six strains from the Leicester Public Health Laboratory culture collection, previously classified as either Branhamella or Neisseria sp, were identified blind. The isolates included four strains each of $N$ meningitidis, $N$ gonorrhoeae, $N$ lactamica and $N$ sicca; one strain each of $N$ flavescens and $N$ subflava; and 28 strains of $B$ catarrhalis. In addition, six clinical isolates obtained from specimens submitted to the laboratory within the period of this study were included.

Accepted for publication 3 September 1987
All strains were cultured on chocolate agar and incubated overnight at $37^{\circ} \mathrm{C}$ in $5 \%$ carbon dioxide. Provisional identification was performed on the basis of colonial appearance, Gram stain; oxidase and catalase reactions. Identification of strains was carried out by standard laboratory methods, using acid production from carbohydrates in serum free sugar slopes, with phenol red as indicator. The carbohydrates used were glucose, sucrose, maltose and lactose, with enzymatic breakdown of O-nitrophenyl3-d-galactopyranoside (ONGP) replacing lactose in some tests. A loopful was scraped off from the chocolate plate culture and inoculated on to the slopes and a few $\mathrm{mm}$ below the surface. The slopes were incubated overnight at $37^{\circ} \mathrm{C}$. A change in the colour of the phenol red indicator from red/pink to pale orange/ yellow was considered to be positive.

For the oxidase test, a portion of a colony was rubbed on a filter paper impregnated with freshly prepared $1 \%$ tetramethyl-p-phenylene diamine hydrochloride. The test was considered to be positive if a purple colour developed within 10 seconds. Catalase activity was detected by the capillary tube method. ${ }^{8}$

Hydrolysis of tributyirin to butiric acid was tested using commercially available tributyrin impregnated tablets. A heavy suspension of the organism was prepared in $0.25 \mathrm{ml}$ of physiological saline. The tablet was dropped into the tube and the tube incubated at $37^{\circ} \mathrm{C}$ for four hours. Positive strains produced a bright yellow colour within no more than two or three hours.

Presumptive $B$ catarrhalis strains were further identified by DNAse production, reduction of nitrate to nitrite, and reduction of nitrite to gas. Production of DNAse was tested for by inoculation of DNAse agar plates (Oxoid CM 321, Oxoid Ltd, Basingstoke, Hampshire) with up to nine tests and one control for each plate. A heavy inoculum was spotted on to the plate and incubated at $37^{\circ} \mathrm{C}$ overnight. The following morning plates were flooded with $1 \mathrm{~N}$ hydrochloric acid, positive strains showed a clear zone around the 
Table Morphological and biochemical characteristics of strains studied

\begin{tabular}{|c|c|c|c|c|c|c|c|}
\hline Species & $N$ menigitidis & $N$ gonorrhoea & N lactamica & $N$ sicca & $N$ subflava & $N$ flavescens & B catarrhalis \\
\hline Morphology & cocci & cocci & cocci & cocci & cocci & cocci & cocci \\
\hline Oxidase & + & + & + & + & + & + & + \\
\hline \multicolumn{5}{|l|}{ Acidification of } & + & + & + \\
\hline Glucose & + & + & + & + & + & - & - \\
\hline Sucrose & - & - & - & + & $+1-$ & - & - \\
\hline Maltose & + & - & + & - & + & - & - \\
\hline Lactose/ONPG & - & - & + & + & - & _- & _- \\
\hline \multicolumn{8}{|l|}{ Reduction of } \\
\hline Nitrate & - & - & - & - & - & - & + \\
\hline Nitrite & $+1-$ & - & + & + & + & + & + \\
\hline Tributyrin hydrolysis & - & - & - & - & $\stackrel{T}{-}$ & $\stackrel{+}{-}$ & + \\
\hline DNAse activity & - & - & - & - & _ & _- & + \\
\hline$\beta$ Lactamase & - & $+1-$ & NT & NT & NT & NT & $+1-$ \\
\hline
\end{tabular}

+ Positive, - Negative, + /-Variable, NT Not tested

growth. Staphylococcus aureus (NCTC strain No 6571) was used as a positive control.

Beta lactamase production was detected by the plate acidometric method. ${ }^{9}$ Colonies were spotted on to a penicillin containing plate, and incubated at $37^{\circ} \mathrm{C}$ for four hours. Production of $\beta$ lactamase was determined by a yellow discolouration of the plate around the area of growth. A $\beta$ lactamase producing strain of Haemophilus influenzae was used as a control.

Nitrate and nitrate reductase activity were tested by emulsifying colonies in 0-25 ml of physiological saline, adding the corresponding tablets and incubating at $37^{\circ} \mathrm{C}$ for four hours, and overnight according to manufacturer's instructions. The reagents (1 drop of dimethylnaphthylamine and 1 drop of sulfanilic acid solution) were added at the end of the incubation period, and all tubes showing a red/pink colour were considered to be positive for nitrate reductase activity and negative for nitrite reductase activity.

\section{Results}

Strains were identified as $N$ meningitidis, $N$ gonorrhoeae, $N$ lactamica, $N$ sicca, $N$ subflava, $N$ flavescens and $B$ catarrhalis according to the reactions they produced (table). These results were compared with previous identification results for all strains recovered from the culture collection.

One strain, previously recorded as $N$ meningitidis, failed to produce sugar reactions in maltose and was later identified by an independent worker as $\mathrm{N}$ gonorrhoeae, confirming the results obtained in this study. All strains identified as $B$ catarrhalis by our previous method produced a positve tributyrin reaction, except strain 24 , which remained negative on repeated testing. All strains were found to reduce nitrate to nitrite, and all except two strains (Nos 21 and 23) were DNAse positive. These two stains failed to show nitrite reductase activity and were assumed to belong to the closely related $N$ ovis species.

\section{Discussion}

Although most of the methods for the isolation of $B$ catarrhalis are dependable, they are laborious and may therefore delay the report in a busy laboratory. "Short cuts" have been taken and most microbiology departments rely on a series of negative tests for their identification of Branhamella sp. Hydrolysis of tributyrin is a well recognised step in the identification of $B$ catarrhalis. The test proved consistently reliable in the identification of the strains tested in this study and correctly differentiated $B$ catarrhalis from Neisseria spp. The combination of Gram stain, oxidase and catalase tests, and hydrolysis of tributyrin provide a means of positive "same day" identification of $B$ catarrhalis. This, together with the availability of rapid methods to perform sugar tests, ${ }^{10}$ should enhance the probability of a correct identification within 24 hours of receipt of clinical specimens. The detection of $\beta$ lactamase activity by any of the rapid reported methods, " which are available to most routine diagnostic microbiology departments, should provide the basis for a useful early report and treatment as appropriate.

\section{References}

1 Brooke N, Rivera M, Luman JI, Wallace R. Branhamella catarrhalis as a lower respiratory tract pathogen in patients with chronic lung disease. Arch Intern Med 1986;146:890-3.

2 Lundgren K, Ingvarsson L. Acute otitis media in Sweden. Role of Branhamella catarrhalis and the rationale for choice of antimicrobial therapy. Drugs 1986;31 (Suppl 3):125-31.

3 Brorson I, Axelsson A, Holm SE. Studies on Branhamella catarrhalis (Neisseria catarrhalis) with special reference to maxillary sinusitis. Scand J Infect Dis 1976;8:151-5. 
4 Wardle JK. Branhamella catarrhalis as an indirect pathogen. Drugs 1986;31 (Suppl 3):93-6.

5 Brook I. Direct and indirect pathogenicity of Branhamella catarrhalis. Drugs 1986;31 (Suppl 3):97-102.

6 Doern GV, Morse SA. Branhamella (Neisseria) catarrhalis: criteria for laboratory identification. J Clin Microbiol 1980; II: $193-5$.

7 Riou JY, Buissiere J, Guibourdenche M, Brault G, Carlier JP. Hydrolyse de la tributyrine par les Neisseria et les Branhamella. Ann Microbiologie 1981;132:159-69.

8 MacFaddin J. Biochemical tests for the identification of medical bacteria. 2nd Ed. Baltimore: Williams and Wilkins, 1983.

9 Park CH, Lopez JS, Cook CB. Acidometric agar plate method for ampicillin susceptibility testing of Haemophilus influenzae. Antimicrob Agents Chemother 1978;13:318-20.

10 Young H, Paterson IC, McDonald DR. Rapid carbohydrate utilization test for the identification of Neisseria gonorrhoeae. British Journal of Venereal Disease 1976;52:172-5.

11 Lucas TH, An evaluation of 12 months for the demonstration of penicillinase. J Clin Path 1974;32;1061-5.

Requests for reprints to: Dr J Richards, Department of Microbiology, Institute of Pathology, Newcastle General, Westgate Road, Newcastle upon Tyne NE4 6BE, England. 